\title{
Carbon nanotubes,
}

\section{materials for the future}

László Forró, Département de Physique, EPF-Lausanne, CH-1015 Lausanne, Switzerland

Christian Schönenberger, Institut für Physik, Unversität Basel, Switzerland

$T$ he last decade of the last century in condensed matter physics $I$ has been marked by the revival of carbon-based materials. Besides the conventional forms of carbon, the graphite and the diamond, new forms of carbon have been discovered: fullerenes, carbon nanotubes, carbon onions. Although the parent compound of fullerenes, the $C_{60}$ molecule was discovered in 1985 by Kroto, Smalley and co-workers [1], the full expansion of the activity concerning this material did not truly begin until the mass production of fullerenes was invented by Krätschmer and Huffman [2]. The great euphoria in the fullerene research started with the discovery of "high temperature superconductivity" in 1991, exceeding a critical temperature of $30 \mathrm{~K} \mathrm{[3]} \mathrm{upon} \mathrm{alkali} \mathrm{metal}$ doping. The search for new carbon nanostructures, higher mass fullerenes has strongly motivated chemists and physicists. Sumio Iijima discovered the multi-walled carbon nanotubes in the same year [4], which was considered at the beginning as a giant fullerene. In 1993 the single walled nanotubes were synthesized giving carbon structures of $1.4 \mathrm{~nm}$ in diameter and several microns in length [5]. At the beginning, while the production and purification of these structures were not sufficiently elaborated, the research mainly consisted of "photography", that is of spectacular images obtained by high-resolution transmission electron microscopy (HRTEM). Around 1994 some of these problems were solved, and the study of the physical properties began.

Today, carbon nanotubes are driving scientific research. This field has several important directions in basic research, including chemistry, electronic transport, mechanical and field emission properties. Furthermore, the perspectives for applications are very challenging and exciting. The main avenues of potential applications of carbon nanotubes are: ultimate reinforcement fibers for composites (high strength, high aspect ratio, high thermal and chemical stability);

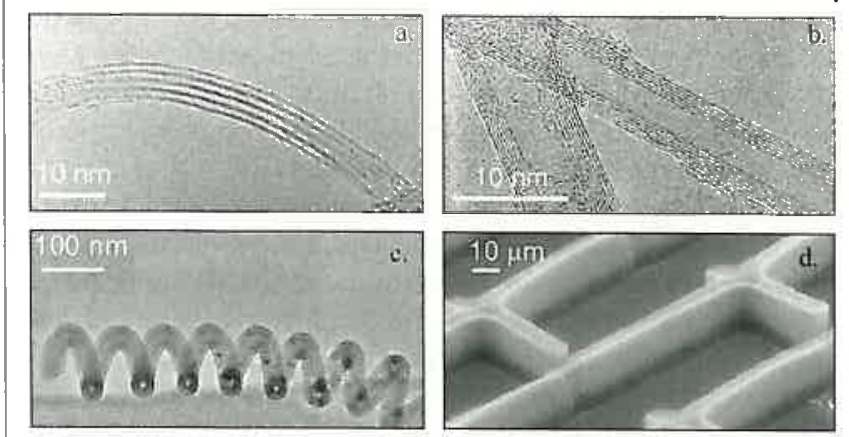

Fig. 1: Montage of electron microscope imags of various carbon nanotubes: a) SWNT rope prepared by laser ablation technique; b) MWNTs synthesized by the arc-discharge method; c) MWNT coil formed in the thermal decomposition of hydrocarbons in the presence of catalytic particles; d) patterns of oriented nanotube bundles grown on pre-structured deposits of catalytic particles by soft lithography. 
conducting nanowires; field emitters (individual nanotube field emitters, large area flat panel displays,); nanotools (tips for Scanning Tunneling, Atomic Force, Magnetic Resonance Force and Scanning Nearfield Optical, Chemical/Biological Force Microscope tips, nanomanipulators, nanotweezers) [6].

In this short article we present these materials, which will certainly rule the condensed matter physics and nanotechnology at the beginning of the 21st century. We highlight a few spectacular results, and show what might be the new directions in this field and beyond.

\section{Materials \\ There are several methods presently used to form carbon nan- otubes. The first is a slightly modified version of the method used for fullerene production, an arc discharge between graphite elec- trodes, allowing a nanotube deposit to accumulate on the cathode. One can form nanotubes of a single rolled up graphene shell (SWNTs) of diameter in the $1 \mathrm{~nm}$ range; and multi-walled nanotubes consisting of several concentrically arranged single- wall carbon tubes nested into each other like a Russian doll. These multishell nanotubes have outer diameters typically in the range of 10-50 nm and are now referred to as multi-walled carbon nan- otubes (MWNTs). Historically, SWNTs were discovered later, after an efficient production method was discovered by Smalley and colleagues, using laser ablation of graphite in the presence of cat- alytic particles. The SWNTs found in the resulting soot are organized into bundles of various diameters. The process of form- ing nanotubes by catalytic decomposition of a carbon containing reaction gas, like acetylene or methane, is extensively used because of two significant advantages. In the first place nanotubes are obtained in large quantity, and at much lower temperatures. This, however, is at the cost of lower perfection, the graphitisation of the tube walls being of poorer quality than in the case of the other two methods. Secondly, the catalyst (for example iron, cobalt or nickel) may be structured on the substrate prior to growth, thus enabling one to grow nanotubes selectively just where one wants to have them. This has enabled the growth of structured "nanobrushes" consisting of vertically aligned nan- otubes at high density. Presently, nanotubes can be grown to a length exceeding 100 micrometers, yielding fibers with a very} high-aspect ratio.

Nature forms carbon nanotubes in various forms, but our understanding of their microscopic growth mechanism is incomplete. For example, via thermal decomposition of hydrocarbons, one can magically grow nanotubes in the form of "telephone cords" or "nanosprings" (potentially suitable for nanomechanical applications), but the understanding of their synthesis is only at an empirical level. The montage of figure 1 illustrates the characteristic forms and shapes of various carbon nanotubes mentioned above.

One has to mention that although these carbon nanostructures are very attractive and their production seems to be simple, they are never prepared in a pure form. The first step in their study is always technological: their purification. This is especially true for SWNTs, which are severely contaminated with magnetic particles from the catalyst. The purity of the arc-discharge fabricated MWNTs is much better, since magnetic materials are not used in their production. Never- theless they have to be separated from graphitic flakes, polyhedral particles and amorphous carbon present in the raw soot.

\section{Electronic properties}

One can view carbon nanotubes as giant conjugated molecular wires with a conjugation length corresponding to the whole length of the tube. In order to understand their electronic structure, we have to start with graphene, a single sheet of graphite. Carbon has four valence electrons of which three are strongly bound to neighbor atoms giving graphene its very high in-plane rigidity. The fourth electron is delocalised and shared by all the atoms, thus allowing for electronic current transport. However, because of its particular structure graphene is electronically between a semiconductor and a metal. It is a semimetal or a "zerogap" semiconductor.

This peculiarity renders the electronic states very sensitive to additional boundary conditions, such as that created by a single shell of a carbon nanotube. A stationary electron wave can only develop, if the circumference of the tube is a multiple of the electron wavelength. This condition removes the zero gap property of graphene and turns a nanotubes into either a true metal or a semiconductor, depending on how the graphene sheet is rolled up, in other words, depending on its helicity. (For MWNTs one expects a more complicated situation, because of a possible additional electronic coupling between adjacent shells). The helicity gives a fascinating richness for the engineering of electronic properties of SWNTs. However, for the time being, we cannot control neither the diameter nor nanotubes' helicity during the synthesis, and at the present this "richness" is rather a drawback than an advantage. For each nanotube one has to find out first its conduction characteristics. In order words, we do not study what we want, but what we get.

The electronic properties of one-dimensional (1d) conductors have generated much interest. The reason for this excitement lies in their very rich phase diagram and the prediction that in a 1d system the Coulomb interaction should lead to a strongly correlated electron gas, called a Luttinger liquid instead of the weakly interacting quasi-particles described as a Fermi-liquid in conventional metals. This issue is still controversial. There are experimental results both for SWNTs and MWNTs, which speak in favour of either exotic Luttinger-liquid or conventional Fermiliquid behaviours.

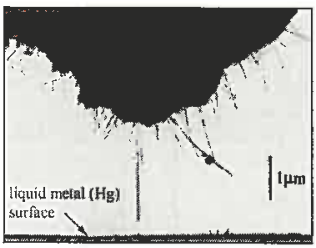

a)

b)

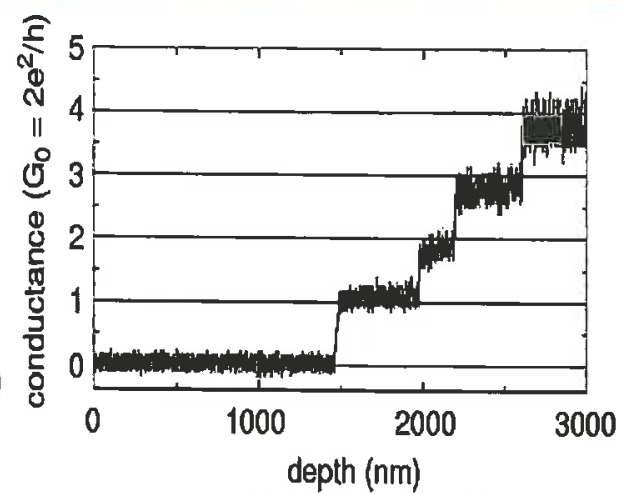

Fig. 2: a) Electron microscope image of an ensemble of MWNTs mounted on a motion stage realized by de Heer and coworkers at Georgia Tech. The MWNT sticking out most is progressively dipped into liquid mercury, which serves as a second electrode. As the individual nanotubes enter the metal, they contribute $G_{0}=2 e^{2} / h$ to the overall quantum conductance, which appears to be ballistic, i.e. independent of length on each plateau. 
A perfect metallic nanotube with uncorrelated electrons, is supposed to be a ballistic conductor, i.e. the best (normal electron) conductor an engineer can dream of, only surpassed by a superconductor. If an electron is injected from a contact into a ballistic wire with ideal contacts, the electron will emerge with certainty at the drain contact. There is no backscattering in the wire, which is the source of intrinsic electric resistance and leads to Ohm's law. For a perfect ballistic tube theory predicts not one, but the existence of two propagating eigenmodes independent of the diameter. The electric conductance (the inverse of the resistance) is then expected to be twice the fundamental conductance unit $\mathrm{G}_{0}=2 \mathrm{e}^{2} / \mathrm{h}=1 / 13 \mathrm{k} \Omega$. Note, the resistance is not zero, as it would be for a superconductor but in contrast to classical resistors and to Ohm's law, the resistance is independent of the length of the wire. Data suggesting that MWNTs are indeed ballistic conductors even at room temperature are highlighted in fig. 2 , although the observed conductance quantum appears to be $\mathrm{G}_{0}$.

Our own investigations have proven that studying electric transport in MWNTs is somewhat similar to studying transport in a large diameter SWNT using lithographically deposited metal contacts in various configurations to connect electrical wires to the tubes (Figure 3). The current mainly flows on the external cylinder, the nanotube core solely acting as a mechanical support for the electrically active outermost shell. (Note, this were no longer true, if we could find a way to contact the core, or even to selectively address inner shells). MWNTs have certain specific advantages over SWNTs: their large diameter favors low-ohmic contacts, because of the larger contact area. Furthermore, the large diameter of MWNTs enables one to investigate quantum-interference phenomena in a magnetic field. The most profound quantum-interference effect is the Aharonov-Bohm (AB) effect that not only reveals that electrons are waves, but also demonstrates that the vector potential not the magnetic field plays a basic role. For the study of this phenomenon, a magnetic field of several Tesla is applied along the nanotube axis. Our electrical resistance

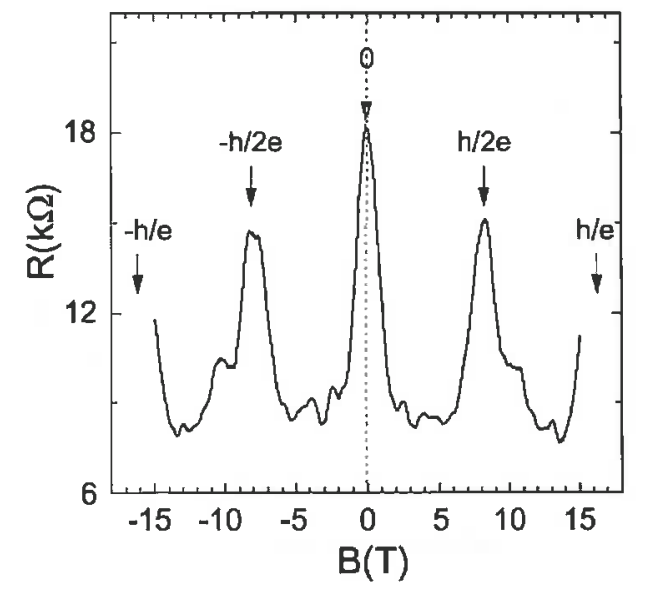

Fig. 4: Electrical resistance $R$ as a function of magnetic field $B$ of a MWNT aligned parallel to $B$. The resistance oscillation is due to the Aharonov-Bohm effect. Arrows denote the resistance maxima corresponding to multiples of $h / 2 \mathrm{e}$ of the magnetic flux through the nanotube outer perimeter, thus indicating that the current flows in the outer most shell.

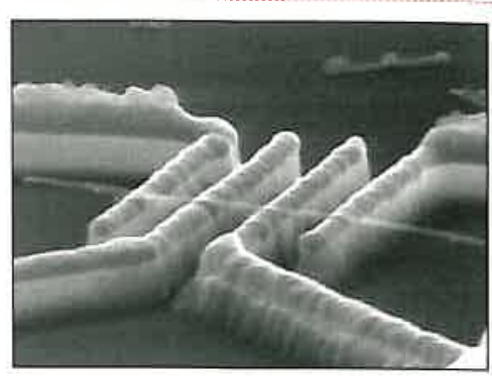

Fig. 3: Scanning Electron Microscopy image of an individual multiwalled carbon nanotube contacted by four electric leads for resistivity measurements. In order to check the influence of the substrate, the $\mathrm{SiO}_{2}$ was etched away from below the nanotube.

measurements showed pronounced oscillations with a period of $\mathrm{h} / 2 \mathrm{e}, \mathrm{h}$ being Planck's constant, and e the electronic charge (Fig. 4). The oscillations are associated with the "weak localization", a quantum-mechanical manifestation of coherent backscattering of electrons, which arises from interference contributions adding up constructively in zero field. Backscattering is thereby enhanced, leading to a resistance larger than the classical Drude resistance. This observation has given compelling evidence that the phase coherence length, can exceed the circumference of the tube. But because the $h / 2 \mathrm{e}$ period (as opposed to $\mathrm{h} / \mathrm{e}$ ) requires backscattering on the scale of the diameter of the MWNT, this implies that these nanotubes are not ballistic, but rather diffusive. Nevertheless, most scattering processes are elastic, i.e. the coherence of electron waves is maintained over a large distance.

In our opinion contradictory results, ballistic contra diffusive transport, Luttinger contra Fermi liquid behavior, do not mean that one experiment is right and the other is wrong, but rather show us that we still do not have control of all experimental parameters, and that more decisive results are yet to come.

\section{Elastic properties}

The carbon atoms of a single sheet of graphite form a planar honeycomb lattice in which each atom is connected via a strong

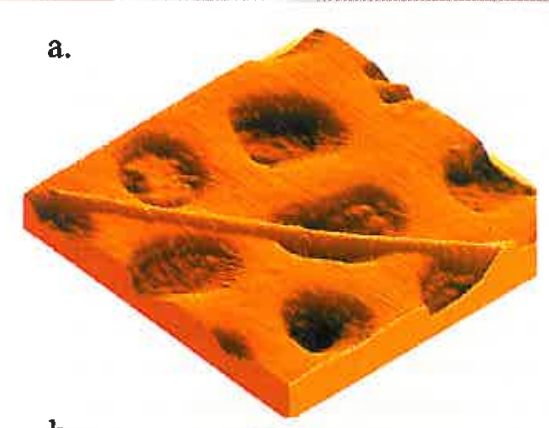

b.

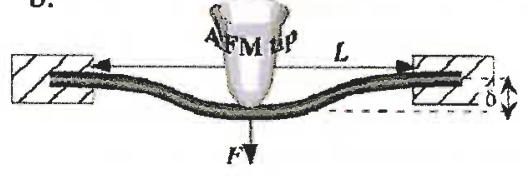

Fig. 5: (a) 3D rendering of an AFM image of a SWNT bundle (or a single MWNT), adhered to an alumina ultrafiltration membrane, leading to a clamped beam configuration for mechanical testing. (b) Schematic representation of the measurement: the AFM tip applies a load, $F$, to the portion with a suspended length of $L$, and the maximum deflection $d$ at the center of the beam is directly recorded from the topographic image; $d$ versus $F$ is proportional to the Young's modulus of the nanotube. 


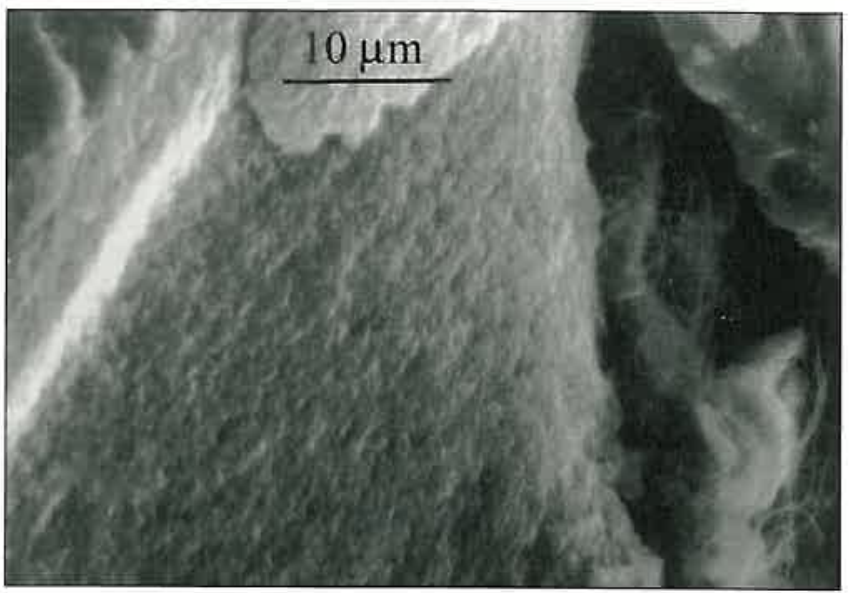

Fig. 6: SEM image of the surface of a fractured Aluminium/ MWNT composite. The central part of the image shows a homogenously dispersed nanotubes in the Al matrix. (Courtesy of R. Schaller, EPFL).

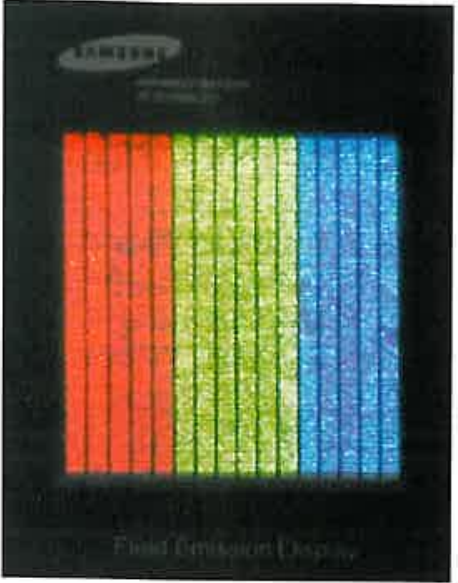

Fig. 7: A prototype of a carbon nanotube based display (Samsung Display Technology, courtesy of Y. Choi). chemical bond to three neighbour atoms. The basal-plane elastic modulus of graphite is one of the largest of any known material. For this reason, carbon nanotubes are expected to be the ultimate high-strength fibres. In our laboratory in Lausanne, we developed a simple method for measuring the mechanical properties of single nanotubes. The technique involves depositing nanotubes from a suspension in a suitable liquid onto well-polished alumina ultrafiltration membranes with a pore size of about $200 \mathrm{~nm}$ (See fig. 5). Carbon nanotubes strongly adhere to alumina, but occasionally span the pores by chance. The deflection of such a supported tube is then deduced from AFM images recorded at various normal loading forces. The measured deflection is inversely proportional to the Young's modulus. It is found to be approximately $0.8 \mathrm{TPa}$ for arc-discharge grown nanotubes, while for the catalytically grown tubes a much lower - by one to two orders of magnitudes modulus was found. This result demonstrates that only highly ordered and well graphitised nanotubes have stiffness comparable to graphite. In contrast, MWNTs grown by catalytic decomposition still contain many defects.

Besides their high strength nanotubes behave magically with respect to high loads. If the applied force exceeds the bending strength, a MWNT first bends over surprisingly large angles, start to ripple on the compressed side and eventually develops kinks, as well. The amazing thing is that all these deformations are elastic, i.e. disappear completely if the load is removed. If one would employ nanotubes as mechanical springs, these springs would be very stiff for small loads, but would turn into soft ones for larger loads allowing for longer extensions without breaking. One could then dream of making objects which after severe deformations relax into their initial form once the load is released.

All these features render nanotubes as very attractive reinforcement fibres for high strength composites. The technological impacts of light and strong structural materials would be enormous. There are reports on epoxy/nanotube, polymer/nanotube composites that have interesting mechanical properties, even though the problem of efficient load transfer to nanotubes has not been yet solved. In our laboratory in Lausanne, the main effort is the development of metal/nanotube composites (fig 6).

\section{Carbon Nanotube Emitters}

Field emission results from the tunneling of electrons from a metal tip into vacuum under application of a strong electric field. The small diameter and high aspect ratio of carbon nanotubes is very favorable for field emission. Even for moderate voltages, a strong electric field develops at the free end of supported nanotubes because of their sharpness. This was observed by de Heer and co-workers at EPFL in 1995. He also immediately realized that these field emitters must be superior to conventional sources and might find their way into all kind of applications, most importantly flat-panel displays. It is remarkable that after only five years Samsung actually realized a very bright colour display, which will be shortly commercialised using this technology (Figure 7).

Studying the field emission properties of MWNT, Bonard and co-workers at EPFL have noticed that together with electrons light is emitted, as well. This luminescence is induced by the electron field emission since it is not detected without applied potential. The typical experimental set-up for electron/light emission is shown in Fig. 8 for an individual nanotube. This light emission occurs in the visible part of the spectrum, and can sometimes be seen with the naked eye.

MWNTs in a configuration shown in fig 8, a single nanotube attached to a wire, also represents an excellent scanning probe tip for AFM and STM studies. Furthermore, due to the light emitting property, it can find application in Scanning Near-field Optical Microscopy, as well.

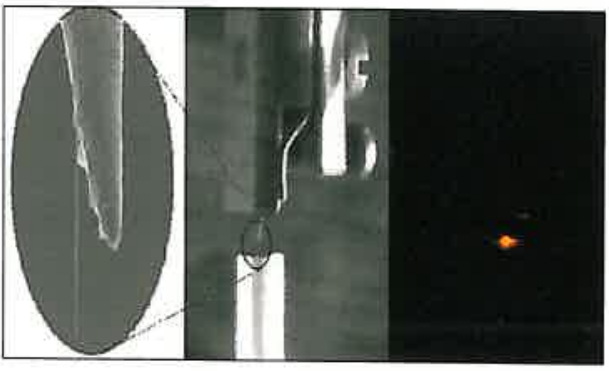

Fig. 8: SEM images a SWNT mounted on a gold wire and photograph of the experimental set-up for field/light emission studies. The emitted red light from the nanotube tip is visible with the naked eye. 


\section{Conclusions}

The future for nanotube looks very bright: Nanotubes are interesting model systems for fundamental studies of one-dimensional systems, but they are equally well (or even more) attractive for applied researchers and industry due to the wide variety of their potential applications. They offer lot of creativity in material preparation. Besides the variety of different structures illustrated in fig. 1, one can fill the hollow core with semiconducting metallic, or ferromagnetic materials etc. Along these lines, the latest "breaking news" is a single wall nanotube filled with $\mathrm{C}_{60}$ molecules (fig. 9). When a few years ago David Luzzi from the University of Pennsylvania first reported such "peapods", one thought of an "exotic bird", very nice, but without any importance for future studies. Today, several laboratories have developed methods for complete filling SWNTs with fullerenes, raising the possibility of creating an almost one-dimensional superconducting wire of $\mathrm{C}_{60}$ inside of a nanotube. A further advantage of this method is that even endohedral fullerenes (containing an atom inside their cages) can be introduced into the nanotubes, e.g. La@C82.

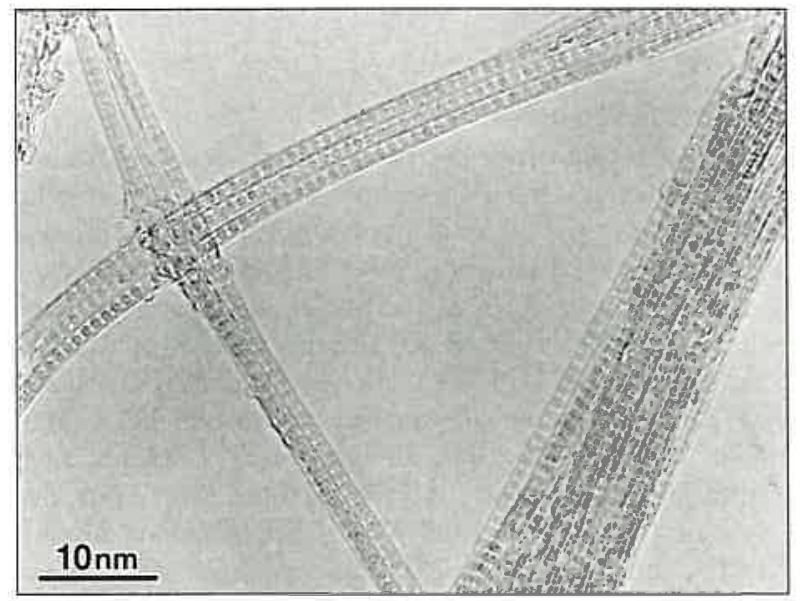

Fig. 8: SEM images a SWNT mounted on a gold wire and photograph of the experimental set-up for field/light emission studies. The emitted red light from the nanotube tip is visible with naked eyes.

Furthermore, since nanotubes are very user-friendly, very robust, they can also act as excellent model systems for learning manipulation at a nanometer scale, which is the scale of biological macromolecules like DNA, microtubules and proteins. For example, the method for measuring the Young's modulus of SWNT ropes was directly applied to measure the same property for individual microtubules in Lausanne, or the electronic conduction of DNA in Basel (group of C. Schönenberger), Delft (group of C. Dekker), and in Orsay (group of H. Bouchiat).

\section{References}

[1] H.W. Kroto, J. R. Heath, S. C. O'Brien, R. F. Curl \& R. E. Smalley Nature 318, 162 (1985).

[2] W. Krätschmer, L. D. Lamb, K. Foristopoulos \& D. R. Huffman Nature 347,354 (1990).

[3] R.C. Haddon et al., Nature 350, 320 (1991).

[4] lijima, S. Nature 354, 56 (1991).

[5] Bethune, D.S., et al. Nature 363, 605 (1993).

[6] for an extensive review on the field see, M.S. Dresselhaus, G. Dresselhaus, and Ph. Avouris, Carbon Nanotubes (Springer Verlag, 2001.) 